\title{
Amplitude and rotational speed control of variable length pendulum by periodic input
}

\author{
Hidekazu Kajiwara $\left(\mathbb{D} \cdot\right.$ Manabu Aoyagi ${ }^{(B)}$
}

Received: 20 July 2020/Accepted: 16 December 2020/Published online: 11 January 2021

(C) The Author(s) 2021

\begin{abstract}
In this paper, a control law to stabilize the amplitude or rotational speed of a variable length pendulum to a desired value by periodically changing the position of the center of gravity is proposed. First, the motion of the pendulum oscillating around a lower equilibrium point is analyzed using the averaging method, and a first-order differential equation for the amplitude of the pendulum is derived. Subsequently, using the derived equation of motion, a control law is designed to control the amplitude of the pendulum to the desired value. Similarly, the motion of a pendulum rotating continuously around the rotation axis is analyzed, the first-order differential equation for the angular velocity of the pendulum is derived, and then a control law of the rotational speed is designed. The derived nonlinear feedback control law consists of the amplitude, angle, and angular velocity of the pendulum in the case of amplitude control, and in the case of rotational speed control, the rotational velocity and angular acceleration of the pendulum. Finally, by using the proposed control method, it is shown that the
\end{abstract}

Supplementary Information The online version contains supplementary material available at (https://doi.org/10.1007/ s11012-020-01299-8) contains supplementary material, which is available to authorized users.

H. Kajiwara $(\bowtie) \cdot$ M. Aoyagi

Graduate School of Engineering, Muroran Institute of

Technology, 27-1 Mizumoto-cho,

Muroran, Hokkaido 050-8585, Japan

e-mail: kajiwara@mmm.muroran-it.ac.jp amplitude and rotational speed of the pendulum can be controlled to the desired values.

Keywords Variable length pendulum · Amplitude control $\cdot$ Rotational speed control $\cdot$ Averaging method

\section{Introduction}

It may be possible to achieve control objectives by applying a periodic input at an appropriate timing to a system that oscillates periodically. For example, it is known that swing pumping can be achieved by changing the position of its center of gravity at twice its natural frequency [1]. Swing pumping has attracted great research attention in the past, and various pumping strategies have been proposed [2-4]. In most previous studies, swing motion has been modeled and analyzed as a variable length pendulum (VLP). Bartucceli et al. [5] investigated heteroclinic and subharmonic bifurcations of VLP using the Melnikov method. Pinsky et al. [6] and Zevin et al. [7] performed qualitative analysis of periodic solutions and their stability for an undamped pendulum with periodically varying length and arbitrary excitation amplitude. Seyranian et al. [8] performed stability analysis of the lower vertical position of a damped pendulum with arbitrary periodic excitation function. Belyakov et al. [9] derived asymptotic expressions for the boundaries 
of the instability domain near resonant frequencies using the averaging method. Akulenko et al. [10] investigated periodic boundary value problems that arise in analyzing the stability of the lower equilibrium point of VLP. In the above studies, the swing model is a one-mass plane pendulum of variable length. In $[11,12]$, the swing model is a weightless rod with two lumped masses, one of which is fixed on the rod and the other slides along it within bounded limits. Aslanov et al. [11] proposed a control law of swing excitation and damping, and showed the asymptotic stability of the system based on a Lyapunov function. Furthermore, Aslanov [12] derived the averaged equation for the amplitude of the pendulum by applying the averaging method to the motion of the pendulum around the lower equilibrium, and proved the asymptotic stability of the averaged system based on a very simple Lyapunov function. In [13-16], the analytical model of the swing is the oscillating physical pendulum induced by a continuous motion of an auxiliary mass. Szyszkowski et al. [13] studied the damping effects in an oscillating physical pendulum induced by continuous motion of an auxiliary mass, and identified the main parameters affecting the damping properties of the pendulum mass system. It was shown that continuous damping can be achieved if the mass motion is synchronized with the pendulum rotation. Octavio et al. [14] proposed a control law plus gravity compensation for active vibration damping in a frictionless physical pendulum with moving mass. The control law can damp out the oscillations of the pendulum by using the moving mass as an active damper. Gandino et al. [15] presented the experimental study of damping in a time-varying inertia pendulum. The system consists of a disk travelling along an oscillating pendulum. The analytical model of the pendulum was introduced and an equivalent damping ratio was estimated by applying energy considerations. Markeev et al. [16] investigated the orbital stability of the periodic motion of the pendulum when the point mass at which the collisions are assumed to be perfectly elastic can collide with the other end of the rod, and determined stability and instability domains in the space of two dimensionless parameters of the system. In conventional studies of VLP, many researchers investigated the stability and parameter dependence of periodic solutions. However, a method to stabilize the amplitude of VLP to a desired value has been seldom investigated. Control approaches based on the energy based control have been proposed as a feedback control for pendular system. Fradkov [17] proposed a method for stabilizing a general Hamiltonian systems to the specified energy level based on the speed-gradient approach. Shiriaev et al. [18] proposed an algorithm ensuring global attractivity of the upright (unstable) equilibrium of a pendulum, based on the variable structure system-version of the energy-speedgradient method. Xin et al. [19] showed that it is difficult to avoid singular points in the controller of VLP designed by using the conventional energy-based control approach proposed in [20, 21], in which the total mechanical energy of the system is controlled. Furthermore, they designed a controller free of singular points by proposing a new Lyapunov function which shapes a part of the total mechanical energy including the kinetic energy of rotation and the potential energy of VLP, and showed their method can stabilize the motion of VLP to a desired swing motion with given desired energy and length of the pendulum by numerical simulations. In [22-26], the motion of the spring pendulum is analyzed as a VLP, and the dynamic response of a harmonically and kinematically excited spring pendulum is studied. In those studies, the transient and steady-state response of resonant/non-resonant states are investigated using the multiple scale method.

Previous studies of VLPs have focused mainly on their motion analysis. The purpose of this research is to design a control law to stabilize the amplitude or rotational speed of a VLP to a desired value by periodically changing the position of the center of gravity.

The rest of this paper is organized as follows. Section 2 presents a mathematical model and the dynamics of a VLP. Section 3 analyzes the motion of a VLP around the lower equilibrium using the averaging method, and derives the control law that stabilizes the amplitude of the VLP to a desired value. Section 4 analyzes the motion of the VLP around the rotation axis using the averaging method, and derives the control law that stabilizes the rotational speed of the VLP to the desired value. Sections 5 and 6 present numerical simulation and experimental results obtained using a real machine that validates the effectiveness of the proposed control law. Finally, Sect. 7 concludes the paper. 


\section{Model and dynamics of variable length pendulum}

In this paper, we design a feedback control law that can control the motion of the pendulum by periodically varying the length of the pendulum. In this section, we derive the equation of motion of the VLP that used when designing the control input.

The mathematical model of the VLP is shown in Fig. 1 , where $\theta$ is the angle to the $y$-axis; $m$ is the pendulum mass; $l$ is the length measured from the fulcrum $O$ to mass $m$; and $f(t)$ is the force acting on the mass. This model is a weightless rod with a point mass sliding along the rod. The kinetic $(T)$, potential $(U)$ energies of the VLP are described as follows:

$T=\frac{1}{2} m l^{2} \dot{\theta}^{2}+\frac{1}{2} m l^{2}$,

$U=-m g l \cos \theta$,

where $g$ is the gravitational acceleration.

The equations of motion are derived from Lagrange's equations of the second kind:

$\frac{d}{d t}\left(\frac{\partial L}{\partial \dot{\theta}}\right)-\frac{\partial L}{\partial \theta}=-d \dot{\theta}$,

$\frac{d}{d t}\left(\frac{\partial L}{\partial i}\right)-\frac{\partial L}{\partial l}=f(t)$,

where $L=T-U$ denotes the Lagrangian, and $d$ is the damping coefficient.

Substituting (1)(2) into (3)(4), the following equations of motion for VLP can be obtained:

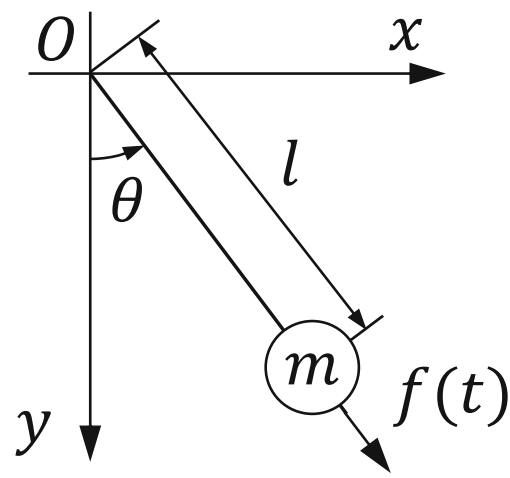

Fig. 1 Mathematical model of VLP $m l^{2} \ddot{\theta}+d \dot{\theta}+2 m l \ddot{\theta}+m g l \sin \theta=0$,

$m \ddot{l}-m l \dot{\theta}^{2}-m g \cos \theta=f$.

The dimensionless form of the above equations is

$(1-\alpha)^{2} \theta^{\prime \prime}+d_{1} \theta^{\prime}-2(1-\alpha) \alpha^{\prime} \theta^{\prime}+(1-\alpha) \sin \theta=0$,

$\alpha^{\prime \prime}-(1-\alpha) \theta^{\prime 2}-\cos \theta=\bar{f}$,

where dimensionless time $\tau=\omega_{0} t, l=l_{0}(1-\alpha), \bar{f}=$ $-f(t) / m l_{0} \omega_{0}^{2}, d_{1}=d / m l_{0}^{2} \omega_{0}, \omega_{0}=\sqrt{g / l_{0}}$.

In this system, it is assumed that a servo system in which $\alpha$ is controlled by $\bar{f}$ is incorporated. At this time, $\bar{f}$ is expressed as follows:

$\bar{f}=-(1-\alpha) \theta^{\prime 2}-\cos \theta+K_{\mathrm{f} 1}\left(\alpha_{\mathrm{d}}-\alpha\right)-K_{\mathrm{f} 2} \alpha^{\prime}$,

where $K_{\mathrm{f} 1}, K_{\mathrm{f} 2}$ represents the proportional and derivative gains of the PD control gain, and $\alpha_{d}$ is a desired value.

When $\bar{f}$ is configured as in the above equation, $\alpha$ follows the $\alpha_{d}$ by setting $K_{\mathrm{f} 1}, K_{\mathrm{f} 2}$ appropriately. In the following, we assume that $\alpha$ is directly controllable.

Let us now consider controlling the pendulum by slightly varying the position of the point mass as follows:

$\alpha=\varepsilon^{2} \bar{\alpha}(0<\varepsilon \ll 1)$,

where $\bar{\alpha}$ is of the order $O(1)$.

Substituting (10) into (7) and omitting the term of $O\left(\varepsilon^{4}\right)$, the following equation can be obtained:

$$
\begin{aligned}
\theta^{\prime \prime}+\sin \theta & =\varepsilon h_{1}\left(\theta^{\prime \prime}, \theta^{\prime}, \theta, \bar{\alpha}^{\prime}, \bar{\alpha}\right) \\
& =\varepsilon\left[2 \varepsilon \bar{\alpha} \theta^{\prime \prime}-\varepsilon^{2} d_{2} \theta^{\prime}+2 \varepsilon \bar{\alpha}^{\prime} \theta^{\prime}+\varepsilon \bar{\alpha} \sin \theta\right],
\end{aligned}
$$

where we assume that $\varepsilon^{3} d_{2}=d_{1}$ and $d_{2}$ is of the order $O(1)$.

In the next section, the equation of motion is approximated according to the motion state of the pendulum, and then the control input $\bar{\alpha}$ is designed.

\section{Amplitude control law design of VLP}

In this section, we design a control law to stabilize the amplitude of a VLP oscillating around the lower equilibrium point to a desired value. First, we analyze the periodic motion of the pendulum by using the averaging method [27]. 
We assume that the pendulum oscillates near a stable equilibrium point and approximate the trigonometric function up to the third order term of the Taylor series:

$\sin \theta=\theta-\varepsilon \delta \theta^{3}$,

where $\varepsilon \delta=1 / 6$.

Substituting (12) into (11), the following equation can be obtained:

$$
\begin{aligned}
\theta^{\prime \prime}+\theta & =\varepsilon h_{2}\left(\theta^{\prime \prime}, \theta^{\prime}, \theta, \bar{\alpha}^{\prime}, \bar{\alpha}\right) \\
& =\varepsilon\left[2 \varepsilon \bar{\alpha} \theta^{\prime \prime}-\varepsilon^{2} d_{2} \theta^{\prime}+2 \varepsilon \bar{\alpha}^{\prime} \theta^{\prime}+\varepsilon \bar{\alpha} \theta-\varepsilon^{2} \delta \bar{\alpha} \theta^{3}+\delta \theta^{3}\right] .
\end{aligned}
$$

Let us now consider replacing $\theta, \theta^{\prime}$ by $\theta_{1}, \theta_{2}$ as follows:

$\theta_{1}=\theta$,

$\theta_{2}=\dot{\theta}$

By using the above relation, (13) can be converted into a system of two first-order differential equations as follows:

$\theta_{1}^{\prime}=\theta_{2}$,

$\theta_{2}^{\prime}=-\theta_{1}+\varepsilon h_{2}\left(\theta_{2}^{\prime}, \theta_{2}, \theta_{1}, \bar{\alpha}^{\prime}, \bar{\alpha}\right)$.

If $\varepsilon=0$, the solutions of the above equations are expressed as follows:

$\theta_{1}=a \sin (\tau+\phi)$,

$\theta_{2}=a \cos (\tau+\phi)$.

From the above solutions, we assume the solutions for $\varepsilon \neq 0$ as follows:

$\theta_{1}=a(\tau) \sin [\tau+\phi(\tau)][a(\tau)>0]$,

$\theta_{2}=a(\tau) \cos [\tau+\phi(\tau)]$,

where $a(\tau)$ and $\phi(\tau)$ are assumed to be slowly varying variables.

Substituting the time derivative of (20) and (21) into (16), the following relation can be obtained:

$a^{\prime} \sin (\tau+\phi)+a \phi^{\prime} \cos (\tau+\phi)=0$.

Substituting (20)(21) and the time derivative of (21) into (17), the following equation can be obtained:

$$
\begin{aligned}
& a^{\prime} \cos (\tau+\phi)-a \phi^{\prime} \sin (\tau+\phi) \\
& =\varepsilon h_{2}\left(\theta_{2}^{\prime}, \theta_{2}, \theta_{1}, \bar{\alpha}^{\prime}, \bar{\alpha}\right) .
\end{aligned}
$$

Solving (22) and (23) for $a^{\prime}, \phi^{\prime}$, the following equations can be obtained:

$a^{\prime}=\varepsilon h_{2}\left(\theta_{2}^{\prime}, \theta_{2}, \theta_{1}, \bar{\alpha}^{\prime}, \bar{\alpha}\right) \cos (\tau+\phi)$,

$a \phi^{\prime}=-\varepsilon h_{2}\left(\theta_{2}^{\prime}, \theta_{2}, \theta_{1}, \bar{\alpha}^{\prime}, \bar{\alpha}\right) \sin (t+\phi)$.

In (13), let us now consider changing $\bar{\alpha}$ at twice the natural frequency of VLP as follows:

$$
\begin{aligned}
\bar{\alpha} & =u(\tau) \sin (\tau+\phi) \cos (\tau+\phi) \\
& =\frac{u}{2} \sin (2 \tau+2 \phi) .
\end{aligned}
$$

Substituting (20)(21) and (26) and the time derivatives of (21)(26) into the right side of (24)(25), and then time averaging for the one period, the following equations can be obtained:

$a^{\prime}=-\frac{\varepsilon^{2}}{2}\left(\varepsilon d_{2}-\frac{3}{4} u\right) a$,

$\phi^{\prime}=-\frac{3}{8} \varepsilon \delta a^{2}-\frac{1}{4} \varepsilon^{2} u^{\prime}$.

In (27), if $a=0$ then $a^{\prime}=0$, and we can see that $a$ cannot be controlled by $u$. Furthermore, if $u$ is as follows, then $a^{\prime}>0$, and $a$ increases monotonically:

$u>\frac{4}{3} \varepsilon d_{2}$.

Dividing both sides of (27) by $a$ and converting the variable as $b=\ln a$, the following equation can be obtained:

$b^{\prime}=-\frac{1}{2} \varepsilon^{3} d_{2}+\frac{3}{8} \varepsilon^{2} u$.

Here, let us now consider $u$ as follows:

$u=K_{p}\left(b_{d}-b\right)+K_{i} \int_{0}^{\tau}\left(b_{d}-b\right) d \bar{\tau}\left(b_{d}=\right.$ const $)$,

where $b_{d}$ is a desired value.

Substituting (31) into (30) and then differentiating the resulting equation with respect to time, the following equation is obtained: 
$b^{\prime \prime}+\frac{3}{8} \varepsilon^{2} K_{\mathrm{p}} b^{\prime}+\frac{3}{8} \varepsilon^{2} K_{\mathrm{i}} b=\frac{3}{8} \varepsilon^{2} K_{\mathrm{i}} b_{\mathrm{d}}$.

By choosing the values of $K_{p}$ and $K_{i}$ so that $b$ becomes asymptotically stable, $b=b_{\mathrm{d}}$ as time $\tau \rightarrow \infty$. Therefore, if $b_{\mathrm{d}}=\ln a_{\mathrm{d}}$, we can see that $a=$ $a_{\mathrm{d}}$ when $b=b_{\mathrm{d}}$.

Changing variables $b, b_{d}$ to $a, a_{d}$, respectively, in (31), the following equation is obtained:

$u=K_{\mathrm{p}} \ln \left(\frac{a_{\mathrm{d}}}{a}\right)+K_{\mathrm{i}} \int_{0}^{\tau} \ln \left(\frac{a_{\mathrm{d}}}{a}\right) d \bar{\tau}\left(a_{\mathrm{d}}=\mathrm{const}\right)$.

Finally, the control input $\bar{\alpha}$ that stabilizes the amplitude of VLP to $a_{\mathrm{d}}$ is expressed as follows:

$\bar{\alpha}=\left[K_{\mathrm{p}} \ln \left(\frac{a_{\mathrm{d}}}{a}\right)+K_{\mathrm{i}} \int_{0}^{\tau} \ln \left(\frac{a_{\mathrm{d}}}{a}\right) d \bar{\tau}\right] \sin (\tau+\phi) \cos (\tau+\phi)$.

Furthermore, the above equation can be expressed as follows by using the periodic motion of (20)(21) and the relation of (14)(15):

$\bar{\alpha}=\left[K_{\mathrm{p}} \ln \left(\frac{a_{d}}{a}\right)+K_{\mathrm{i}} \int_{0}^{\tau} \ln \left(\frac{a_{d}}{a}\right) d \bar{\tau}\right] K_{\mathrm{AG} 1} \theta \cdot K_{\mathrm{AG} 2} \theta^{\prime}$,

where $K_{\mathrm{AG} 1}$ and $K_{\mathrm{AG} 2}$ are the gains of the automatic gain control for adjusting the amplitude of $\theta$ and $\theta^{\prime}$ to 1 , respectively.

Therefore, the amplitude of the VLP can be controlled by the above control input.

\section{Rotational speed control law design of VLP}

In this section, we design a control law to stabilize the angular velocity of a VLP rotating continuously around the rotation axis to a desired value

Let us now consider replacing $\theta, \theta^{\prime}$ by $\hat{\theta}_{1}, \hat{\theta}_{2}$ as follows:

$\hat{\theta}_{1}=\theta$,

$\hat{\theta}_{2}=\dot{\theta}$

By using the above relation, (11) can be converted into a system of two first-order differential equations as follows: $\hat{\theta}_{1}^{\prime}=\hat{\theta}_{2}$,

$\hat{\theta}_{2}^{\prime}=-\sin \hat{\theta}_{1}+\varepsilon h_{1}\left(\hat{\theta}_{2}^{\prime}, \hat{\theta}_{2}, \hat{\theta}_{1}, \bar{\alpha}^{\prime}, \bar{\alpha}\right)$.

Assuming $\varepsilon=0$ in (39) and multiplying both sides of (39) by $\hat{\theta}_{2}$ and integrating, the following equation is obtained:

$\frac{1}{2} \hat{\theta}_{2}^{2}=\frac{1}{2} \omega^{2}+\cos \hat{\theta}_{1}\left(\right.$ when $\left.\left|\hat{\theta}_{1}\right|=\frac{\pi}{2}, \hat{\theta}_{2}=\omega\right)$.

Solving the above equation for $\hat{\theta}_{2}$, the following is obtained:

$\hat{\theta}_{2}=\omega \sqrt{1+2 \varepsilon^{2} \sigma \cos \hat{\theta}_{1}}$,

where we assume that $\varepsilon^{2} \sigma=1 / \omega^{2}$ and $\sigma$ is of the order $O(1)$.

Expanding (41) with $\varepsilon^{2} \sigma$ and ignoring the highorder terms, the following equation is obtained:

$\hat{\theta}_{2}=\omega+\varepsilon^{2} \sigma \omega \cos \hat{\theta}_{1}$.

In the above equation, the angular velocity comprises a component that is constant over time and a component that changes periodically, and we can predict that the time average of the angular velocity is $\omega$. Therefore, we assume the angle of VLP as follows:

$\hat{\theta}_{1}=\omega \tau+\varepsilon^{2} \sigma \sin (\omega \tau)$.

From the above solutions (42)(43), we assume the solution for $\varepsilon \neq 0$ as follows.

$\hat{\theta}_{1}=\omega(\tau) \tau+\varepsilon^{2} \sigma \sin [\omega(\tau) \tau]$,

$\hat{\theta}_{2}=\omega+\varepsilon^{2} \sigma \omega \cos \hat{\theta}_{1}$,

where $\omega(\tau)$ is assumed to be slowly varying variables.

Furthermore, we assume the following relation from (40):

$\frac{1}{2} \hat{\theta}_{2}^{2}=\frac{1}{2} \omega(\tau)^{2}+\cos \hat{\theta}_{1}\left(\right.$ when $\left.\left|\hat{\theta}_{1}\right|=\frac{\pi}{2}, \hat{\theta}_{2}=\omega\right)$.

Substituting (39) into the time derivative of (46), the following equation can be obtained:

$\omega \omega^{\prime}=\varepsilon h_{1}\left(\hat{\theta}_{2}^{\prime}, \hat{\theta}_{2}, \hat{\theta}_{1}, \bar{\alpha}^{\prime}, \bar{\alpha}\right) \hat{\theta}_{2}$. 
Now, let us now consider to change $\bar{\alpha}$ as follows in (47):

$$
\bar{\alpha}=u(\tau) \sin (\omega \tau) .
$$

Substituting (44)(45)(48) and the time derivative of (45)(48) into (47) and then time averaging for the one period, the following equation is obtained:

$\omega^{\prime}=-\varepsilon^{3} d_{2} \omega+\frac{3}{2} \varepsilon^{2} u$.

From the above equation, we can see that $\omega$ can be controlled by a proportional-integral controller as follows:

$u=K_{\mathrm{p}}\left(\omega_{\mathrm{d}}-\omega\right)+K_{\mathrm{i}} \int_{0}^{\tau}\left(\omega_{\mathrm{d}}-\omega\right) d \bar{\tau}\left(\omega_{\mathrm{d}}=\right.$ const $)$,

where $\omega_{\mathrm{d}}$ is the desired angular velocity.

Finally, the control input $\bar{\alpha}$ that stabilizes the angular velocity of the VLP to $\omega_{\mathrm{d}}$ is expressed as follows:

$\bar{\alpha}=\left[K_{\mathrm{p}}\left(\omega_{\mathrm{d}}-\omega\right)+K_{\mathrm{i}} \int_{0}^{\tau}\left(\omega_{\mathrm{d}}-\omega\right) d \bar{\tau}\right] \sin (\omega t)$.

Here, let us now consider expressing the above equation by the motion of pendulum.

Substituting (44)(45) into the time derivative of (45), the following is obtained:

$$
\begin{aligned}
\hat{\theta}_{2}^{\prime}= & \omega^{\prime}+\varepsilon^{2} \sigma \omega^{\prime} \cos (\omega \tau)-2 \varepsilon^{2} \sigma \cos (\omega \tau) \sin (\omega \tau) \\
& -\sin (\omega \tau) .
\end{aligned}
$$

In (52), because $\hat{\theta}_{2}^{\prime}=O(1)$, the following equation can be obtained:

$\hat{\theta}_{2}^{\prime} \approx-\sin (\omega \tau)$.

In this case, (51) can be expressed as follows by using (53) and the time derivative of (37):

$\bar{\alpha}=-\left[K_{\mathrm{p}}\left(\omega_{\mathrm{d}}-\omega\right)+K_{\mathrm{i}} \int_{0}^{\tau}\left(\omega_{\mathrm{d}}-\omega\right) d \bar{\tau}\right] K_{\mathrm{AG} 3} \theta^{\prime \prime}$,

where $K_{\mathrm{AG} 3}$ is the gain of the automatic gain control for adjusting the amplitude of $\theta^{\prime \prime}$ to 1 .

Therefore, the angular velocity of the VLP can be controlled by the above control input.

\section{Numerical simulation}

In this section, we show the result validated by numerical simulations to show whether the VLP can be controlled by the periodic input. In the simulation, we used (7) (8) (9) to simulate the motion of the VLP and set the parameters of the VLP and the initial conditions to $\left(d, K_{\mathrm{f} 1}, K_{\mathrm{f} 2}\right)=(0.001,5000,100)$ and $\left(\theta, \theta^{\prime}\right)=(0.1,0)$.

The desired value $\alpha_{\mathrm{d}}$ for amplitude control consists of the following based on (10) (35):

$$
\begin{aligned}
\alpha_{\mathrm{d}}= & \varepsilon^{2}\left[K_{\mathrm{p}} \ln \left(\frac{a_{d}}{a}\right)+K_{\mathrm{i}} \int_{0}^{\tau} \ln \left(\frac{a_{d}}{a}\right) d \bar{\tau}\right] K_{\mathrm{AG} 1} \theta \\
& \cdot K_{\mathrm{AG} 2} \theta^{\prime} .
\end{aligned}
$$

In the above equation, $K_{\mathrm{AG} 1}$ and $K_{\mathrm{AG} 2}$ are derived by measuring the respective amplitude of every half period and by calculating the inverse of the amplitude as shown below:

$K_{\mathrm{AG} 1}=\frac{1}{|\theta|_{\max }}, \quad K_{\mathrm{AG} 2}=\frac{1}{\left|\theta^{\prime}\right|_{\max }}$.

Figure 2 shows the time response of $\theta, \alpha$. The control gains are $\left(\varepsilon, K_{\mathrm{p}}, K_{\mathrm{i}}\right)=(0.1,4.0,0.001)$. We
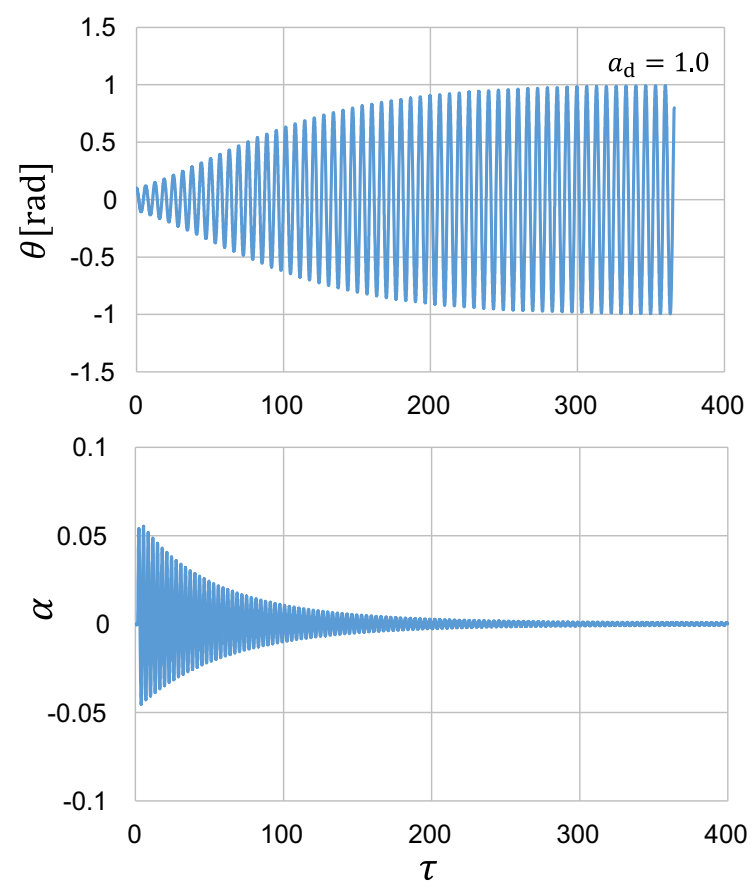

Fig. 2 Time response $\theta, \alpha$ 
can see that the amplitude of the VLP converges to the desired value $\alpha_{d}=1$.

Figure 3 shows the time responses of $|\theta|_{\max }$ and $a$ calculated from the averaging Eq. (27) and its input (33) when $a_{\mathrm{d}}$ is varied from 0.5 to 3.0. We can see that the error between $|\theta|_{\max }$ and $a$ increases as $a_{\mathrm{d}}$ increases. We can also see that the amplitude of the VLP can be controlled over a wide range using the designed control input. Next, we describe the simulation results of the rotation speed control of the VLP.

The desired value $\alpha_{\mathrm{d}}$ for rotation control consists of the following based on (10)(54):

$\alpha_{\mathrm{d}}=-\varepsilon^{2}\left[K_{\mathrm{p}}\left(\omega_{\mathrm{d}}-\theta^{\prime}\right)+K_{\mathrm{i}} \int_{0}^{\tau}\left(\omega_{\mathrm{d}}-\theta^{\prime}\right) d \bar{\tau}\right] K_{\mathrm{AG} 3} \theta^{\prime \prime}$.

In the above equation, $K_{\mathrm{AG} 3}$ is calculated as follows:

$K_{\mathrm{AG} 3}=\frac{1}{\left|\theta^{\prime \prime}\right|_{\max }}$,

where $\left|\theta^{\prime \prime}\right|_{\max }$ is the amplitude of every half period.

Figure 3 shows the time response of $\theta^{\prime}, \alpha$. The control gain and the initial value are $\left(\varepsilon, K_{\mathrm{p}}, K_{\mathrm{i}}\right)=$ $(0.1,2.0,0.002)$ and $\left(\theta, \theta^{\prime}\right)=(\pi / 2,1.5)$, respectively. We can see that the rotational speed of the VLP converges to the desired value $\omega_{d}=10$.

Figure 4 shows the time responses of $\theta^{\prime}$ and $\omega$ calculated from the averaging Eq. (49) and its input (50) when $\omega_{\mathrm{d}}$ is varied from 5 to 15 . We can see $\theta^{\prime}$ converges near the desired value and $\omega$ expresses the average value of $\theta^{\prime}$. (Figure 5)

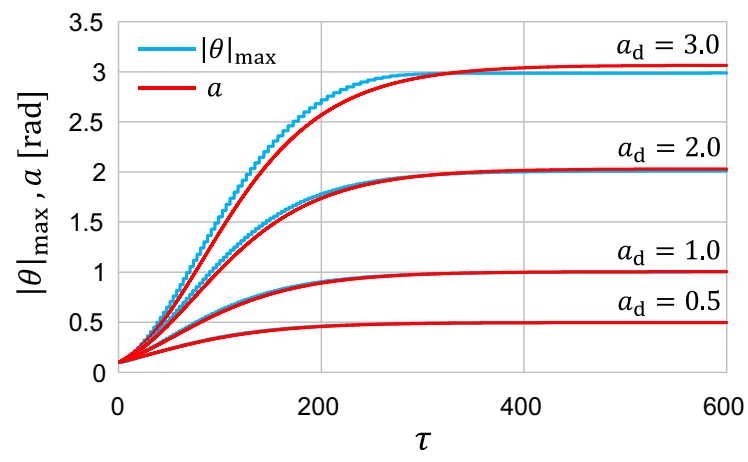

Fig. 3 Time response $|\theta|_{\max }, a$
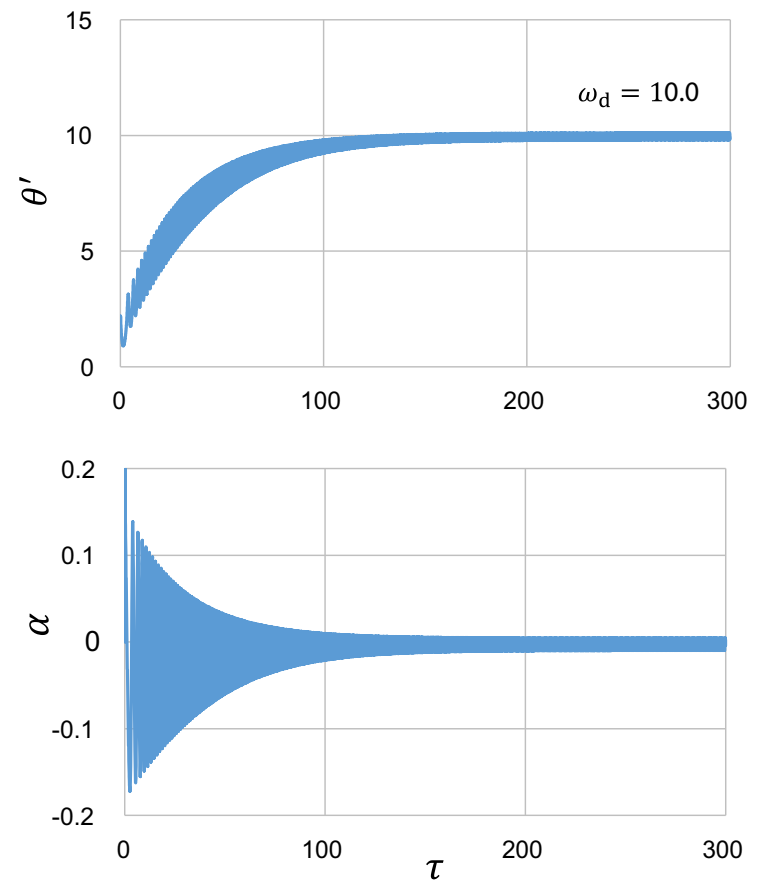

Fig. 4 Time response $\theta^{\prime}, \alpha$

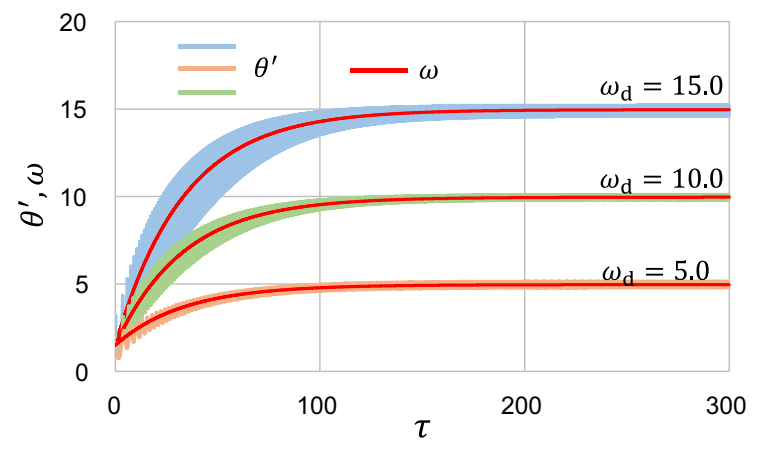

Fig. 5 Time response $\theta^{\prime}, \omega$

\section{Experiment}

\subsection{Experimental system}

The VLP experimental system is shown in Fig. 6. The pendulum is fixed to the rotary shaft and rotates with the rotary shaft. The pendulum consists of an RC servomotor and a rack \& pinion mechanism. The weight can be moved up and down as the motor rotates. Consequently, the position of the center of gravity of the pendulum is changed. The rotation angle of the pendulum is measured by the rotary encoder attached in the rotary shaft. The power and rotation 


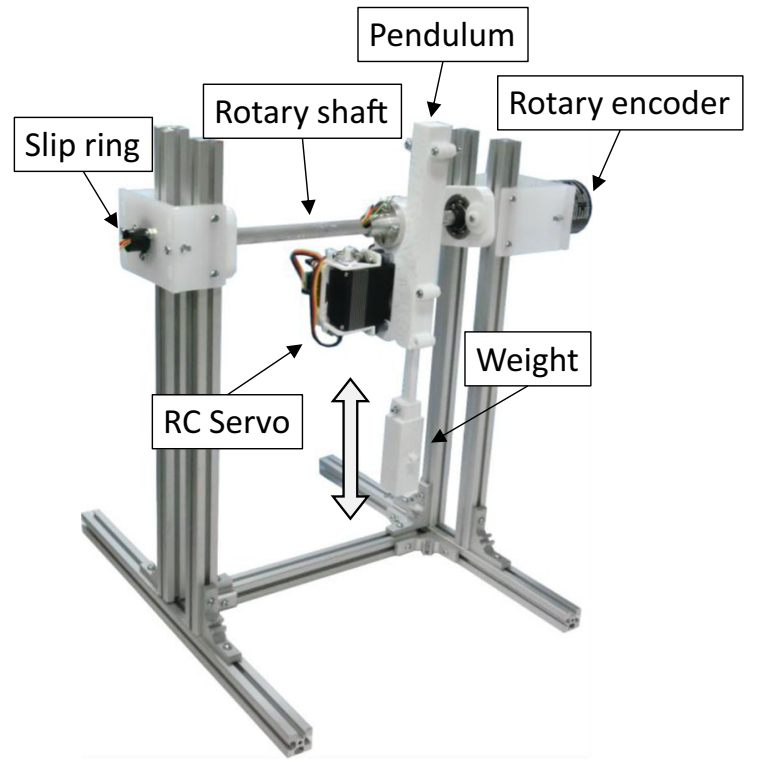

Fig. 6 Experimental system of VLP

angle command to the RC servomotor are given through the slip ring. The parameters of the pendulum used in the experiment are listed in Table 1. These parameters are estimated by assuming that the pendulum is a simple pendulum with friction.

\subsection{Experimental result}

As shown in Sect. 3, when the amplitude of the VLP is 0 , the motion of the VLP cannot be controlled by changing the center of gravity. In this system, the weight is attached in order to move in a direction slightly inclined from the vertical axis to generate the initial vibration in the resting condition of the pendulum. Therefore, even if the pendulum is in the resting condition, a small oscillation can be generated in the horizontal direction by changing the position of the weight.

The control procedure used in the amplitude control experiment is shown below:

Table 1 Parameters of VLP in the experiment

$m=0.210[\mathrm{~kg}]$

$l_{0}=0.126[\mathrm{~m}]$

$d=5.92 \cdot 10^{-4}[\mathrm{Nms} / \mathrm{rad}]$

$$
\begin{aligned}
0 \leq t<4[s]: & l_{0} \alpha_{d} \\
= & 0.015 \sin \left(\omega_{0} t+\pi\right)\left(\omega_{0}=8.82\right),
\end{aligned}
$$

$$
\begin{aligned}
4 \leq t: & l_{0} \alpha_{d} \\
= & \varepsilon^{2}\left[K_{\mathrm{p}} \ln \left(\frac{a_{\mathrm{d}}}{a^{*}}\right)+K_{\mathrm{i}} \int_{0}^{t} \ln \left(\frac{a_{\mathrm{d}}}{a^{*}}\right) d \bar{t}\right] K_{\mathrm{AG} 1} \theta \\
& \cdot K_{\mathrm{AG} 2} \dot{\theta}
\end{aligned}
$$

where $a^{*}$ is derived by measuring the respective amplitude of every half period and by calculating the moving average per second.

First, the initial vibration is generated by changing the weight position at the natural frequency. Subsequently, the amplitude is controlled by switching to the derived control law.

Figure 7 shows that the VLP can be effectively stabilized at the desired value of $a_{\mathrm{d}}=1.0$ with the initial conditions $(\theta, \dot{\theta})=(0,0)$ and the control gain $\left(\varepsilon, K_{\mathrm{p}}, K_{\mathrm{i}}\right)=(0.01,3,1)$.

Figure 8 shows the time responses of $a^{*}$ when $a_{\mathrm{d}}$ is varied from 0.25 to 2.8 . We can see that the amplitude of the VLP can be controlled over a wide range using the derived control input.

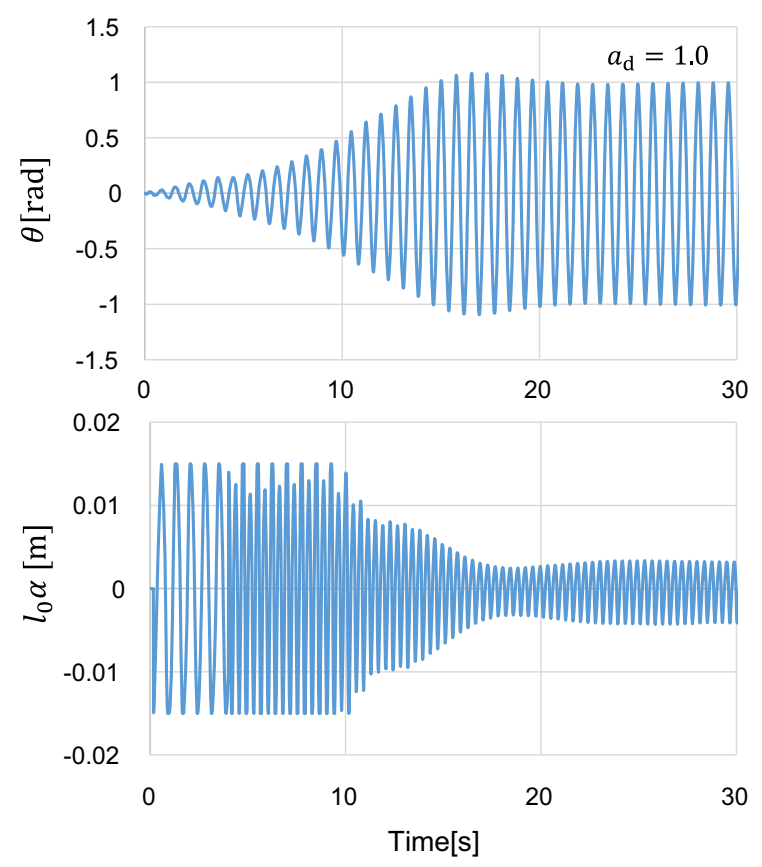

Fig. 7 Time response $\theta, l_{0} \alpha$ 


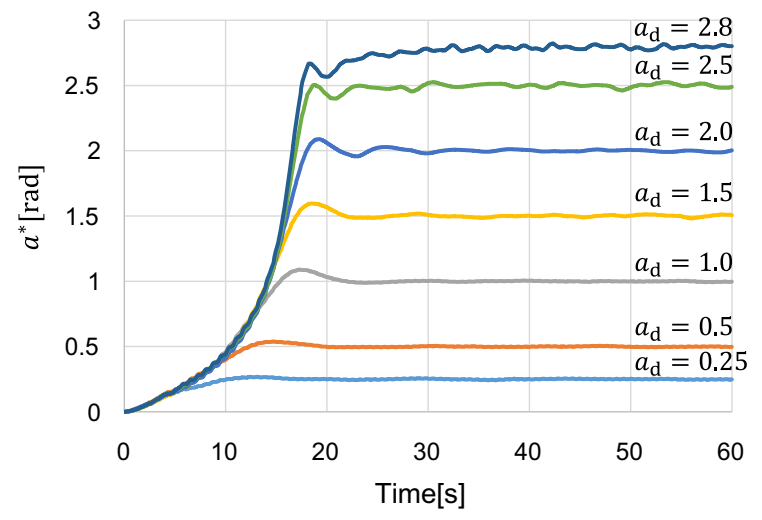

Fig. 8 Time response of $a^{*}$

The rotational speed control results of the VLP are described as follows.

The control procedure used in the rotational speed control experiment is shown below:

$0 \leq t<4[s]: \quad l_{0} \alpha_{d}=0.015 \sin \left(\omega_{0} t+\pi\right)\left(\omega_{0}=8.82\right)$,

$4 \leq t$ and $\omega^{*}<3[\mathrm{rad} / \mathrm{s}]: \quad l_{0} \alpha_{d}=0.03 K_{\mathrm{AG} 1} \theta \cdot K_{\mathrm{AG} 2} \dot{\theta}$,

$4 \leq t$ and $3 \leq \omega^{*}: \quad l_{0} \alpha_{d}=-\varepsilon^{2}\left[K_{\mathrm{p}}\left(\omega_{\mathrm{d}}-\omega^{*}\right)+K_{\mathrm{i}} \int_{0}^{t}\left(\omega_{\mathrm{d}}-\omega^{*}\right) d t\right] \quad K_{\mathrm{AG} 3} \ddot{\theta}$,

where $\omega^{*}$ is the moving average per second of $\dot{\theta}$. In the time interval $0 \leq t<4$, the initial vibration is generated by using resonance. In the time interval $4 \leq t$, the amplitude is monotonously increased by moving the weight at twice the natural angular frequency. Subsequently, when $3 \leq \omega^{*}$, the angular velocity is controlled by switching to the derived control law.

Figure 9 shows that the VLP is controlled at $\omega_{\mathrm{d}}=$ 15 with the initial conditions $(\theta, \dot{\theta})=(0,0)$ and the control gain $\left(\varepsilon, K_{\mathrm{p}}, K_{\mathrm{i}}\right)=(0.01,0.2,0.05)$. We can see that the average angular velocity is controlled to the desired value after the amplitude of the VLP is increased monotonically.

Figure 10 shows the time responses of $\omega^{*}$ when $\omega_{\mathrm{d}}$ is varied from 10 to 20 with the initial conditions $(\theta, \dot{\theta}) \approx(1.0,0)$. The desired values and gain are $\left(\omega_{\mathrm{d}}, \varepsilon, K_{\mathrm{p}}, K_{\mathrm{i}}\right)=(10,0.01,0.3,0.1),\left(\omega_{\mathrm{d}} \varepsilon, K_{\mathrm{p}}, K_{\mathrm{i}}\right)=$ $(15,0.01,0.2,0.05),\left(\omega_{\mathrm{d}}, \varepsilon, K_{\mathrm{p}}, K_{\mathrm{i}}\right)=(20,0.01,0.1,0.02)$.

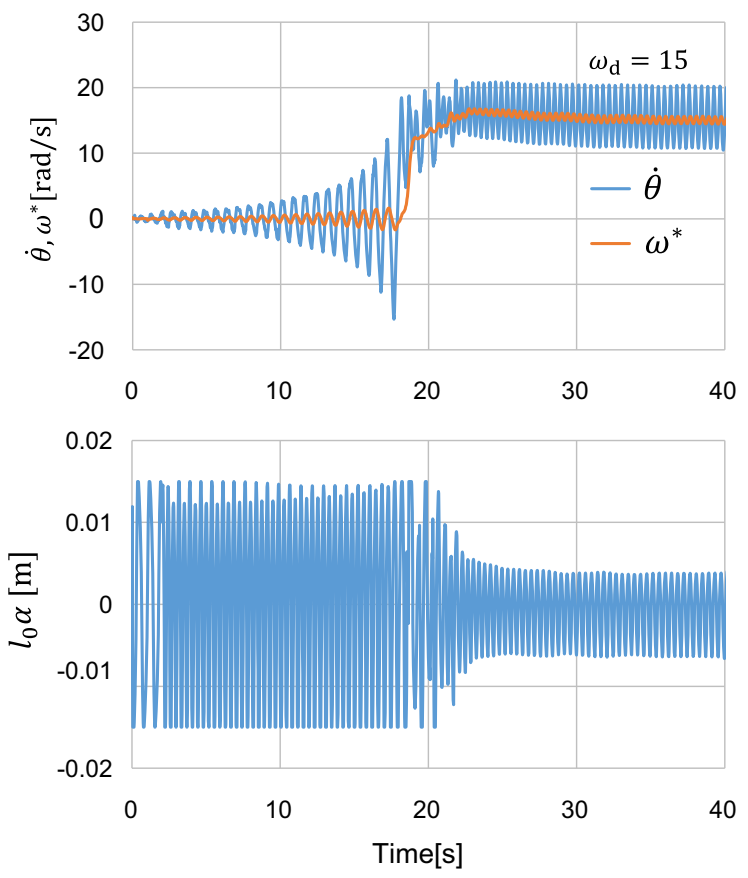

Fig. 9 Time response of $\dot{\theta}, \omega^{*}, l_{0} \alpha$

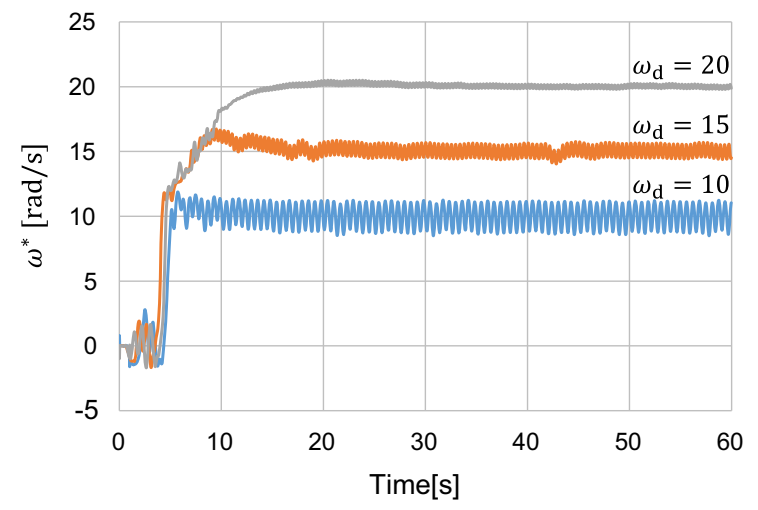

Fig. 10 Time response of $\omega^{*}$

We can see that the average angular velocity of the VLP converges to the desired value.

\section{Conclusion}

In this study, when the position of the center of gravity of the pendulum was moved periodically, the oscillating motion of the pendulum around the lower equilibrium point and the motion of continuous rotational motion were analyzed using the averaging 
method, and the averaged equation of the pendulum was derived. A control law stabilizing the amplitude and angular velocity of the VLP to the desired values was designed using the averaged equation. Finally, using the proposed control method, it was shown that the amplitude and rotational speed of the pendulum can be controlled to the desired values. Similar to the studies on VLPs, the motion of a pendulum with a vertically oscillating support (also called parametrically forced pendulum) has been investigated in previous studies [28-34]. In [35], a comparison between the motions of a parametrically forced pendulum and VLP was reported, and the differences and similarities between the two systems were demonstrated. As the two systems demonstrate similar characteristics, our proposed control approach for a VLP can be considered equally applicable to a parametrically forced pendulum.

\section{Compliance with ethical standards}

Conflict of interest The authors declare that they have no conflict of interest.

Open Access This article is licensed under a Creative Commons Attribution 4.0 International License, which permits use, sharing, adaptation, distribution and reproduction in any medium or format, as long as you give appropriate credit to the original author(s) and the source, provide a link to the Creative Commons licence, and indicate if changes were made. The images or other third party material in this article are included in the article's Creative Commons licence, unless indicated otherwise in a credit line to the material. If material is not included in the article's Creative Commons licence and your intended use is not permitted by statutory regulation or exceeds the permitted use, you will need to obtain permission directly from the copyright holder. To view a copy of this licence, visit http://creativecommons.org/licenses/by/4.0/.

\section{References}

1. Arnold VI (1989) Mathematical methods of classical mechanics, 2nd edn. Springer, New York, p 119

2. Case WB (1996) The pumping of a swing from the standing position. Am J Phys 64(3):215-220

3. Wirkus S, Rand R, Ruina A (1998) How to pump a swing. College Math J. 29(4):266-275

4. Piccoli B, Kulkarni J (2005) Pumping a swing by standing and squatting: do children pump time optimally? IEEE Control Syst Mag 25(4):48-56

5. Bartuccelli M, Christiansen PL, Muto V, Soerensen MP, Pedersen NF (1988) Chaotic behaviour of a pendulum with variable length. Il Nuovo Cimento B 100(2):229-249
6. Pinsky MA, Zevin AA (1999) Oscillations of a pendulum with a periodically varying length and a model of swing. Int J Non-Linear Mech 34(1):105-109

7. Zevin AA, Filonenko LA (2007) A qualitative investigation of the oscillations of a pendulum with a periodically varying length and a mathematical model of a swing. J Appl Math Mech 71(6):892-904

8. Seyranian AP (2004) The swing: parametric resonance. J Appl Math Mech 68(5):757-764

9. Belyakov AO, Seyranian AP, Luongo A (2009) Dynamics of the pendulum with periodically varying length. Physica D 238(16):1589-1597

10. Akulenko LD, Nesterov SV (2009) The stability of the equilibrium of a pendulum of variable length. J Appl Math Mech 73(6):642-647

11. Aslanov VS, Bezglasnyi SP (2012) Stability and instability of controlled motions of a two-mass pendulum of variable length. Mech Solid 47(3):285-297

12. Aslanov VS (2019) Stability of a pendulum with a moving mass: the averaging method. J Sound Vib 445:261-269

13. Szyszkowski W, Stilling DSD (2005) On damping properties of a frictionless physical pendulum with a moving mass. Int J Non Lin Mech 40(5):669-681

14. Gutiérrez-Frias OO, Martínez-García JC, Moctezuma RAG (2009) PD control for vibration attenuation in a physical pendulum with moving mass. Math Probl Eng 2009:179724

15. Gandino E, Marchesiello S, Bellino A, Fasana A, Garibaldi L (2014) Damping effects induced by a mass moving along a pendulum. Shock Vib 2014:314527

16. Markeev AP (2017) On stability in a case of oscillations of a pendulum with a mobile point mass. J Appl Math Mech 81(4):262-269

17. Fradkov AL (1996) Swinging control of nonlinear oscillations. Int J Control 64(6):1189-1202

18. Shiriaev AS, Egeland O, Ludvigsen H, Fradkov AL (2001) VSS-version of energy-based control for swinging up a pendulum. Syst Control Lett 44(1):45-56

19. Xin X, Liu Y (2014) Trajectory tracking control of variable length pendulum by partial energy shaping. Commun Nonlinear Sci Numer Simul 19(5):1544-1556

20. Fantoni I, Lozano R (2001) Non-linear control for underactuated mechanical systems. Springer, New York

21. Kolesnichenko O, Shiriaev AS (2002) Partial stabilization of underactuated Euler-Lagrange systems via a class of feedback transformations. Syst Control Lett 45(2):121-132

22. Starosta R, Sypniewska-Kamińska G, Awrejcewicz J (2011) Asymptotic analysis of kinematically excited dynamical systems near resonances. Nonlinear Dyn 68:459-469

23. Starosta R, Sypniewska-Kamińska G, Awrejcewicz J (2011) Parametric and external resonances in kinematically and externally excited nonlinear spring pendulum. Int J Bifurcat and Chaos 21(6):3013-3021

24. Awrejcewicz J, Starosta R, Sypniewska-Kamińska G (2016) Stationary and transient resonant response of a spring pendulum. Procedia IUTAM 19:201-208

25. Sypniewska-Kamińska G, Starosta R, Awrejcewicz J (2018) Two approaches in the analytical investigation of the spring pendulum. Vib Phys Syst 29:2018005

26. Sypniewska-Kamińska G, Awrejcewicz J, Kamiński H, Salamon R (2020) Resonance study of spring pendulum based on asymptotic solutions with polynomial 
approximation in quadratic means. Meccanica. https://doi. org/10.1007/s11012-020-01164-8

27. Awrejcewicz J, Krysko AV (2006) Introduction to asymptotic methods. Chapman and Hall/CRC Press, New York

28. Lenci S, Pavlovskaia E, Rega G, Wiercigroch M (2008) Rotating solutions and stability of parametric pendulum by perturbation method. J Sound Vib 310(1-2):243-259

29. Krasil'nikov PS (2012) The non-linear oscillations of a pendulum of variable length on a vibrating base. J Appl Math Mech 76(1):25-35

30. Bulanchuk PO, Petrov AG (2012) The vibrational energy and control of pendulum systems. J Appl Math Mech 76(4):396-404

31. Brzeski P, Perlikowski P, Yanchuk S, Kapitaniak T (2012) The dynamics of the pendulum suspended on the forced Duffing oscillator. J Sound Vib 331(24):5347-5357
32. Aduyenko AA, Amel'kin NI (2015) Resonance rotations of a pendulum with a vibrating suspension. J Appl Math Mech 79(6):531-538

33. Han N, Cao Q (2017) A parametrically excited pendulum with irrational nonlinearity. Int $\mathrm{J}$ Non-Linear Mech 88:122-134

34. Neishtadt AI, Sheng K (2017) Bifurcations of phase portraits of pendulum with vibrating suspension point. Commun Nonlinear Sci Numer Simul 47:71-80

35. Wrighta JA, Bartuccellia M, Gentileb G (2017) Comparisons between the pendulum with varying length and the pendulum with oscillating support. J Math Anal App 449(2):1684-1707

Publisher's Note Springer Nature remains neutral with regard to jurisdictional claims in published maps and institutional affiliations. 